\title{
Engineering iodine-doped carbon dots as dual-modal probes for fluorescence and X-ray CT imaging
}

This article was published in the following Dove Press journal:

International Journal of Nanomedicine

6 November 2015

Number of times this article has been viewed

\author{
Miaomiao Zhangl,* \\ Huixiang Ju $\mathrm{u}^{2, *}$ \\ Li Zhang ${ }^{1, *}$ \\ Mingzhong Sun ${ }^{2}$ \\ Zhongwei Zhou ${ }^{2}$ \\ Zhenyu Dai ${ }^{3}$ \\ Lirong Zhang' \\ Aihua Gong' \\ Chaoyao Wu' \\ Fengyi Du'
}

'School of Medicine, Jiangsu University, Zhenjiang, People's Republic of China; ${ }^{2}$ Department of Clinical Laboratory, Affiliated Yancheng Hospital, School of Medicine, Southeast University, Yancheng, Jiangsu, People's Republic of China; ${ }^{3}$ Radiology Department, Affiliated Yancheng Hospital, School of Medicine, Southeast University, Yancheng, Jiangsu, People's Republic of China

*These authors contributed equally to this work
Correspondence: Huixiang Ju Department of Clinical Laboratory, Affiliated Yancheng Hospital, School of Medicine, Southeast University, 75 Juchang Road, Yancheng, Jiangsu 22400I, People's Republic of China

Tel +865I58I600198

Email 2659661466@qq.com

Fengyi Du

School of Medicine, Jiangsu University, 30I Xuefu Road, Zhengjiang, 212013, People's Republic of China

Email biodfy@ujs.edu.cn

\begin{abstract}
X-ray computed tomography $(\mathrm{CT})$ is the most commonly used imaging technique for noninvasive diagnosis of disease. In order to improve tissue specificity and prevent adverse effects, we report the design and synthesis of iodine-doped carbon dots (I-doped CDs) as efficient CT contrast agents and fluorescence probe by a facile bottom-up hydrothermal carbonization process. The as-prepared I-doped CDs are monodispersed spherical nanoparticles (a diameter of $\sim 2.7 \mathrm{~nm}$ ) with favorable dispersibility and colloidal stability in water. The aqueous solution of I-doped CDs showed wavelength-dependent excitation and stable photoluminescence similar to traditional carbon quantum dots. Importantly, I-doped CDs displayed superior X-ray attenuation properties in vitro and excellent biocompatibility. After intravenous injection, I-doped CDs were distributed throughout the body and excreted by renal clearance. These findings validated that I-doped CDs with high X-ray attenuation potency and favorable photoluminescence show great promise for biomedical research and disease diagnosis.
\end{abstract}

Keywords: carbon dots, contrast agents, iodine-doped, CT imaging

\section{Introduction}

During the past decade, medical imaging (MI) technologies have been emerging as a powerful noninvasive tool to visualize, characterize, and quantify the physiological and pathological process for disease diagnosis and biomedical research. ${ }^{1-3}$ The modalities of MI technologies are currently used in optical imaging, ${ }^{4}$ ultrasound, ${ }^{5}$ magnetic resonance imaging, ${ }^{6}$ computed tomography $(\mathrm{CT}),{ }^{7}$ and positron-emission tomography. ${ }^{8}$ As one of the most commonly used MI technologies, CT offers more spatial anatomic details with high resolution than other imaging modalities. In comparison to other MI technologies, CT provides superior images of electron-dense materials due to X-ray attenuation by tissues. ${ }^{9}$ Therefore, the contrast resolution of CT is easy to distinguish soft tissues from bone tissue based on the distinct X-ray attenuation capacity. Even so, subtle changes of soft tissues, such as lesion, anabasis, and tumor, are difficult to perceive through CT image because these soft tissues have similar X-ray attenuation properties, ranging from 0 to $50 \mathrm{HU} \cdot{ }^{10}$ Consequently, the injection of contrast agents is necessary to achieve accurate and abundant information of region of interest. ${ }^{11}$

Iodinated compounds are widely used as CT contrast agents in clinical applications due to their prominent photoelectric effect. ${ }^{12}$ The cube of the high atomic number $(Z=53)$ endows iodine with favorable $\mathrm{X}$-ray attenuation property through photoelectric effect. ${ }^{13}$ However, iodinated contrast agents, with the help of the blood circulation system, spread throughout body after intravenous injection and are excreted through rapid renal clearance. ${ }^{14}$ The nonspecificity and short circulation restricts their wide applications in vivo. Additionally, inherent properties of iodinated aqueous solution, such as high osmolality and viscosity, sometimes induce serious adverse effect 
related to the excretion pathway. Due to these restrictions, various kinds of nanoparticles as efficient CT contrast agents have been developed to overcome these abovementioned limitations. ${ }^{15,16}$

Carbon quantum dots, emerging as new stars of carbon nanomaterials, have attracted tremendous attention due to their outstanding properties and potential applications. ${ }^{15}$ Especially in biomedical field, favorable water-solubility, good biocompatibility, and facile surface modification enabled their use as a multifunctional nanoplatform for bioimaging, ${ }^{17}$ biosensor, ${ }^{18}$ and targeted drug delivery system. ${ }^{19}$ In previous research studies, we demonstrated that functionalized carbon dots (CDs) could be used as effective fluorescent probes for bioimaging and biolabeling living cells. ${ }^{20,21}$ Meanwhile, as a commonly functionalized strategy, doping CDs with heteroatoms provides an attractive method of effectively tuning their intrinsic properties and exploiting new performance for advanced device applications. ${ }^{22}$ In view of X-ray attenuation and heteroatom-doped strategy, we report a hydrothermal carbonization (HTC) approach for facile preparation of iodine-doped carbon dots (I-doped CDs) as efficient CT contrast agents for the first time. In line with the intensive research, we demonstrated that iodine atoms could be successfully doped into the carbon nanoparticles and that the I-doped CDs possessed similar physicochemical and optical properties of conventional CDs. Compared with traditional iodinated contrast agent, the resultant I-doped CDs exhibited not only unique photoluminescence (PL) and X-ray attenuation property, but also long circulation and passive targeted CT imaging.

\section{Materials and methods Materials}

Glycine and quinine sulfate (98\%, suitable for fluorescence) were purchased from Sigma (New York, NY, USA). Iodixanol was purchased from Hengrui Pharmaceutical Co., Ltd (Lianyungang, Jiangsu, People's Republic of China). 3-(4,5Dimethyl-thiazol-2-yl)-5-(3-carboxymethoxyphenyl)-2-(4sulfophenyl)-2H-tetrazolium (MTS) was purchased from Promega (CellTiter Aqueous One Solution cell proliferation Assay kit, Madison, WI, USA). $\mathrm{NaH}_{2} \mathrm{PO}_{4}, \mathrm{Na}_{2} \mathrm{HPO}_{4}$, and $\mathrm{H}_{2} \mathrm{SO}_{4}$ were obtained from Guangfu Fine Chemical Research Institute (Nankai, Tianjin, People's Republic of China). Fetal bovine serum and Dulbecco's Minimum Essential Medium (DMEM) were purchased from Invitrogen China Limited (Shanghai, People's Republic of China). All chemicals were of analytical grade and were used without further purification.

\section{Synthesis of I-doped CDs}

First, certain amounts of iodixanol and glycine were diluted with $20 \mathrm{~mL}$ water under vigorous stirring to form a transparent homogeneous solution. This solution was transferred into a $50 \mathrm{~mL}$ Teflon-lined stainless steel autoclave and heated at $180^{\circ} \mathrm{C}$ for different periods ( 3 hours). After cooling to room temperature, the reaction mixture was centrifuged at 5,000 rpm for 15 minutes to remove the black precipitates. The brownyellow supernatant was transferred into a dialysis membrane (molecular weight cut-off of 1,000 Da) and was dialyzed against ultrapure water for 4 days to remove residues. The dialysis solution was collected and freeze-dried using a vacuum freeze dryer at $-50^{\circ} \mathrm{C}$ for 48 hours. The I-doped CD powders thus obtained were saved for further characterization.

\section{Instrumentation and characterizations}

The chemical structures of I-doped CDs were analyzed using a Fourier transform infrared (FTIR) spectrometer (Nicolet Nexus 470, GMI, Ramsey, MN, USA). The elemental composition was determined by elemental analysis performed on a CHNS-O analyzer. The morphologies of the I-doped CDs were examined by high-resolution transmission electron microscopy on a JEM-2100 microscope (JEOL, Tokyo, Japan) under an accelerating voltage of $200 \mathrm{kV}$. UV-Vis (ultraviolet-visible) absorption spectra were recorded using a UV-2450 UV-Vis Spectrophotometer (Shimadzu, Kyoto, Japan). PL emission measurements were made using a Cary Eclipse Fluorometer (Varian, Palo Alto, CA, USA). The iodine element of I-doped CDs was quantified by linear calibration with a standard content of previously determined amounts of potassium iodine by inductively-coupled plasma atomic emission spectroscopy.

\section{Measurement of fluorescence quantum yields}

The quantum yield $(\Phi)$ of the as-prepared I-doped CDs was determined by a comparative method. ${ }^{23} \mathrm{~A}$ standard solution of quinine sulfate (reported quantum yield: $54 \%$ ) was prepared by dissolving the substance in $0.1 \mathrm{M} \mathrm{H}_{2} \mathrm{SO}_{4}$ (refractive index $[\eta]=1.33$ ). Aqueous solutions containing varying concentrations of I-doped CDs were prepared by dissolving in distilled water $(\eta=1.33)$. Absorbance of the solutions at the excitation wavelength was measured using a UV-Vis spectrophotometer. PL emission spectra of all the samples were recorded using an FLS920 fluorometer at an excitation wavelength of $360 \mathrm{~nm}$. The samples were then analyzed using the PL spectrometer and the PL emission intensity at the excitation wavelength at which the I-doped CDs and the 
reference showed the same UV absorbance. The quantum yield was calculated using the following equation:

$$
\Phi_{\mathrm{X}}=\Phi_{\mathrm{ST}}\left(\frac{\operatorname{Grad}_{\mathrm{X}}}{\operatorname{Grad}_{\mathrm{ST}}}\right)\left(\frac{\eta_{\mathrm{X}}^{2}}{\eta_{\mathrm{ST}}^{2}}\right)
$$

ST and X denote the standard group and test group, respectively. $\Phi$ is the fluorescence quantum yield. Grad is the gradient from the plot of integrated fluorescence intensity vs absorbance, and $\eta$ is the refractive index of the solvent. To minimize the reabsorption effects, absorbance in the $10 \mathrm{~mm}$ fluorescence cuvette was not allowed to exceed 0.1 at the excitation wavelength.

\section{Cell viability assay and cell imaging}

The cytotoxicity of I-doped CDs was evaluated on A549 and 4T1 cells using the MTS assay according to the protocol provided by the manufacturer. Briefly, these cells were seeded in a 96-well plate at a density of $3 \times 10^{3}$ cells/well and incubated for 24 hours at $37^{\circ} \mathrm{C}$ and $5 \% \mathrm{CO}_{2}$, and then the growth medium was replaced with DMEM containing different concentrations of I-doped CDs. Each sample was prepared in triplicate. After incubation for 24 hours, $20 \mu \mathrm{L}$ MTS solution was added to each well and incubated for 3 hours at $37^{\circ} \mathrm{C}$ under $5 \% \mathrm{CO}_{2}$. The absorbance of each well was measured at $490 \mathrm{~nm}$ using Synergy HT Multi-Mode Microplate Reader (Bio Tek, Winooski, VT, USA). Nontreated cells (in DMEM) were used as control, and the relative cell viability (mean \pm $\mathrm{SD}, \mathrm{n}=3$ ) was expressed as $\mathrm{Abs}_{\text {sample }} / \mathrm{Abs}_{\text {control }} \times 100 \%$.

The A549 and 4T1 cells were used to examine the utility of the newly developed I-doped CDs in live cell imaging. The two cell types were seeded on $15 \mathrm{~mm}$ diameter glass cover-slips placed inside the wells of a 24 -well plate and were cultured for 24 hours at $37^{\circ} \mathrm{C}$ and $5 \% \mathrm{CO}_{2}$ in medium containing $10 \%$ fetal bovine serum. Medium containing I-doped CDs at a concentration of $50 \mathrm{mg} / \mathrm{mL}$ was then added to each well and the cells were incubated for 6 hours at $37^{\circ} \mathrm{C}$ and $5 \% \mathrm{CO}_{2}$. The labeled cells were then washed with phosphate buffer saline (PBS) and fixed with $4 \%$ paraformaldehyde/PBS at room temperature for 10 minutes. After washing the cells, a confocal lasers scanning fluorescence microscope (Zeiss LSM-710, Carl Zeiss Meditec AG, Jena, Germany) was used to observe and record the fluorescence emission from CDs inside the cells.

\section{Hemolysis assay}

Hemolysis assay was carried out according to the procedure reported in the literature with slight modification. ${ }^{24,25}$ In brief, fresh mouse blood stabilized with heparin sodium was kindly prepared by Animal Management Rules of the Ministry of Health of the People's Republic of China and the guidelines for the Care and Use of the Jiangsu University Laboratory Animal Center. The blood was centrifuged (1,200 rpm, 15 minutes) to remove the supernatant and washed with PBS five times to completely remove serum and obtain the mouse red blood cells (MRBCs). Thereafter, the MRBCs were diluted ten times with PBS. The diluted MRBC suspension $(0.1 \mathrm{~mL})$ was transferred into $2 \mathrm{~mL}$ tubes prefilled with $0.9 \mathrm{~mL}$ water (as positive control), $0.9 \mathrm{~mL}$ PBS (as negative control), and $0.9 \mathrm{~mL}$ PBS containing I-doped CDs with different particle concentrations $(50-200 \mu \mathrm{g} / \mathrm{mL})$, respectively. The mixtures were incubated for 2 hours at $37^{\circ} \mathrm{C}$ after a gentle shaking, and centrifuged at $12,000 \mathrm{rpm}$ for 1 minute. Finally, the photographs of the samples were taken and the absorbance of the supernatants (hemoglobin) was measured by a UV-2450 UV-Vis spectrophotometer. The hemolysis percentages of different samples were calculated by dividing the difference in absorbance at $541 \mathrm{~nm}$ between the sample and the negative control by the difference in absorbance at $541 \mathrm{~nm}$ between the positive and negative controls.

\section{In vivo and in vitro $C T$ phantom study}

To study the X-ray attenuation in vitro, I-doped CDs with different concentration ranging from 0 to $0.2 \mathrm{mg} / \mathrm{mL}$ were diluted into $100 \mu \mathrm{L}$ water and added to a 96-well culture plate. CT images of the samples were acquired on a clinical 64-slice multidetector CT scanner (SOMATOM Emotion, Siemens, Bavaria, Munich, Germany). These samples were imaged with the following parameters: tube voltage, $130 \mathrm{kV}$; current intensity, $180 \mathrm{~mA}$; slice thickness, $5.0 \mathrm{~mm}$; scan time, 2.85 seconds.

For CT imaging in vivo, we choose the Sprague Dawley rats as animal model. Animal experiments were approved and performed in accordance with the Animal Management Rules of the Ministry of Health of the People's Republic of China and the guidelines for the Care and Use of the Jiangsu University Laboratory Animal Center. Before the intravenous injection, the samples solution was filtered through sterilized membrane filters (pore size $0.22 \mu \mathrm{m}$ ) and stored at $4^{\circ} \mathrm{C}$ for following experiments. The mice given intravenous injection of $1 \mathrm{~mL}$ I-doped CDs in PBS solution. The CT images of mice injected with I-doped CDs were acquired under the following parameters: tube voltage, $130 \mathrm{kV}$; current intensity, $25 \mathrm{~mA}$.

\section{Histological analysis}

For histological studies, mice were sacrificed 30 days after intravenous injection of I-doped CDs at a dose of $20 \mathrm{mg} / \mathrm{kg}$. 
Rats without the injection of I-doped CDs were used as control groups. Tissues (heart, spleen, liver, lung, and kidney) were harvested from the above two groups (control and test groups). The ex vivo organs were immersed in a buffered solution of 4\% paraformaldehyde for 24 hours, dehydrated, and treated for inclusion in paraffin. The specimen was sectioned serially at $4 \mathrm{~mm}$ thickness and stained with hematoxylin and eosin. The histological sections were observed under an optical microscope.

\section{Results and discussion \\ Preparation and characterization of I-doped CDs}

The successful preparation of I-doped CDs was carried out by using iodixanol as iodine source and glycine as surface passivation agent based on the scheme given in Figure 1. The morphology of the synthesized I-doped CDs was analyzed by transmission electron microscopy. As shown in Figure 2A, the I-doped CDs exhibited uniform dispersion and discrete quasispherical shape without apparent aggregation. Figure 2B shows that the diameter distribution of I-doped CDs matched well with Gaussian fitting curve. The average diameter of I-doped CDs was approximately $2.7 \mathrm{~nm}$, which was determined by statistical analysis of more than 100 particles by using the ImageJ software (National Institutes of Health, Bethesda, MD, USA). Meanwhile, these nanoparticles were amorphous without any lattices, which was consistent with other reports on CDs. ${ }^{20,21}$

\section{The chemical structure and surface composition of the I-doped CDs}

The surface functional groups and composition of the I-doped CDs were investigated using FTIR spectrum and X-ray photoelectron spectroscopy pattern. As shown in Figure 3A, the FTIR pattern exhibited characteristic absorption bonds of $\mathrm{O}-\mathrm{H}$ and $\mathrm{N}-\mathrm{H}$ stretching vibrations of amine groups at $3,420 \mathrm{~cm}^{-1}, \mathrm{C}-\mathrm{H}$ stretching vibrations at $2,940 \mathrm{~cm}^{-1}, \mathrm{C}=\mathrm{C}$ stretching vibrations at $1,660 \mathrm{~cm}^{-1}, \mathrm{C}-\mathrm{N}$ stretching vibrations at $1,410 \mathrm{~cm}^{-1}$, and $\mathrm{C}-\mathrm{H}$ stretching vibrations at $1,070 \mathrm{~cm}^{-1}$. The X-ray photoelectron spectroscopy survey spectra of the as-prepared I-doped CDs (Figure 3B) showed four typical peaks at 619.0, 284.0, 400.0, and $530.6 \mathrm{eV}$, which indicated that the I-doped CDs were mainly composed of iodine, carbon, nitrogen, and oxygen atoms. The highresolution spectrum of $\mathrm{I}_{3 \mathrm{~d} 5}$ revealed the presence of $3_{\mathrm{d} 5 / 2}$ and $3_{\mathrm{d} 3 / 2}$ doublet at 619.6 and $631.1 \mathrm{eV}$, respectively. This suggested that iodine existed in the form of negatively charged iodine. ${ }^{26}$ There were two weak peaks at 617.1 and $628.7 \mathrm{eV}$<smiles>CC(=O)N(CC(O)CN(C(C)=O)c1c(I)c(C(=O)NCC(O)CO)c(I)c(C(=O)NCC(O)CO)c1I)c1c(I)c(C(=O)NCC(O)CO)c(I)c(C(=O)NCC(O)CO)c1I</smiles>

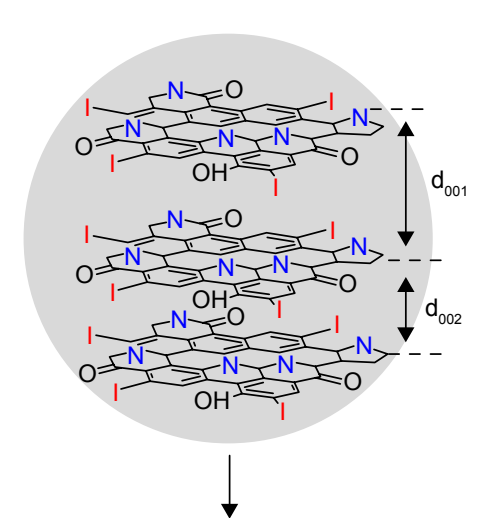

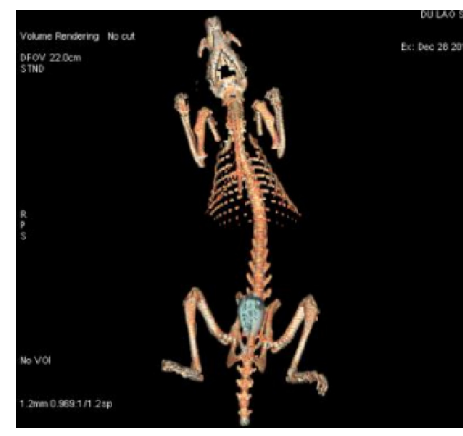

CT imaging

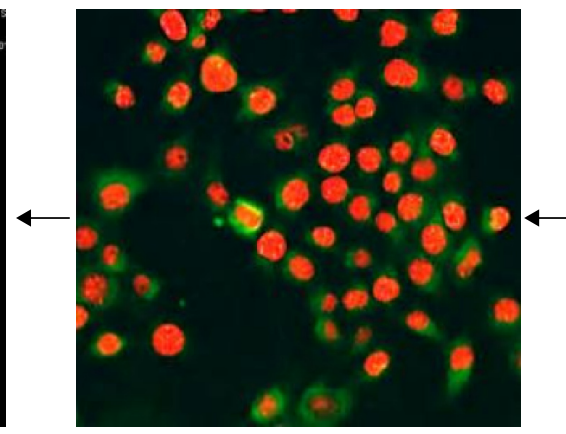

Fluorescence imaging

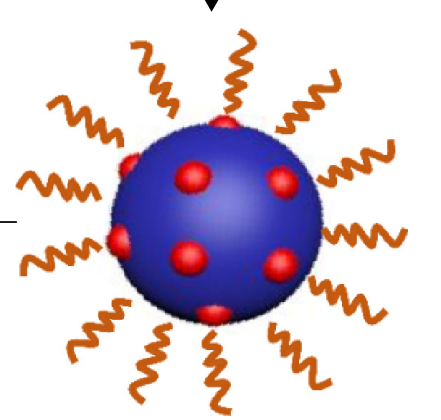

I-doped CDs

Figure I Schematic synthesis of I-doped CDs from the HTC of iodixanol and glycine and following application. Abbreviations: I-doped CDs, iodine-doped carbon dots; HTC, hydrothermal carbonization; CT, computed tomography. 
A

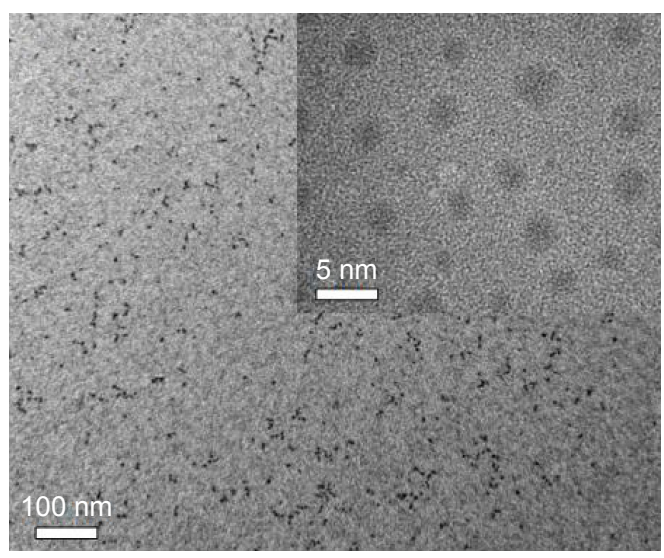

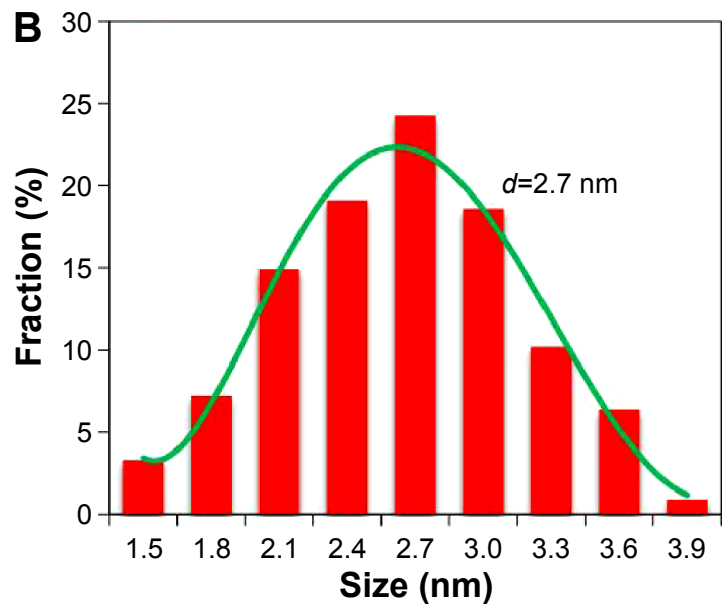

Diameter distribution $=$ Gaussian fitting

Figure 2 Morphology characterization of I-doped CDs.

Notes: (A) Low and high (inset) magnification TEM images. (B) The diameter distribution of prepared I-doped CDs.

Abbreviations: I-doped CDs, iodine-doped carbon dots; TEM, transmission electron microscopy.

which were ascribed to the characteristic bonds of iodine in $\mathrm{I}_{2}{ }^{27}$ During the hydrothermal process, the trace amount of $\mathrm{I}_{2}$ species was probably absorbed on the surface defect sites of the I-doped CD nanoparticles. The high-resolution spectrum of $\mathrm{C}_{1 \mathrm{~S}}$ (Figure 3C) indicated the three main peaks at $283.7,285.3$, and $287.3 \mathrm{eV}$, which were attributed to $\mathrm{C}-\mathrm{C}$, $\mathrm{C}-\mathrm{I}$ and $\mathrm{C}-\mathrm{C}, \mathrm{C}-\mathrm{O}$, respectively. The binding energy peak at $283.7 \mathrm{eV}$ confirmed the graphitic structure (sp2, C-C) of the I-doped CDs. These results revealed that the functional groups of I-doped CDs mainly contained certain iodine and amino groups, plentiful carbonyl, carboxylate, and hydroxyl groups. The presence of these functional groups located at their surface endowed the I-doped CDs with excellent solubility and dispersibility in water.

The crystal structure of the I-doped CDs was investigated by X-ray diffraction. As shown in Figure 4A, there were three diffraction peaks in the I-doped CDs pattern around $13.17^{\circ}, 29.6^{\circ}$, and $42.7^{\circ}$, respectively. The corresponding interlayer spacing (d) was calculated from the Bragg's law (the wavelength of $\mathrm{Cu}-\mathrm{K} \alpha(\lambda)$ is $0.154 \mathrm{~nm}$ ). These diffraction peaks matched well with the characteristic peaks of graphite oxide. As for the main peak, the diffraction peak of I-doped CDs around $13.17^{\circ}\left(\mathrm{d}_{001}=0.671 \mathrm{~nm}\right)$ was similar to the typical diffraction peak $\left(2 \theta=10.6^{\circ},\{001\}\right.$ plane $)$ of graphite oxide, which was attributed to an increase in sp3 layers spacing. During HTC process, many functional groups such as hydroxyl, carbonyl, epoxy, and amino groups were formed and bonded to the edges of basal planes of the crystal structure, inducing an increase in the interlayer spacing. Additionally, the second diffraction peak of I-doped CDs around $29.6^{\circ}\left(\mathrm{d}_{002}=0.301 \mathrm{~nm}\right)$ became stronger and was more similar to the characteristic peaks of graphite $(\{002\}$ planes, $2 \theta=26.5^{\circ}$ ) than graphite oxide. However, compared to the ordered crystal structure of graphite, the upward shift (from $26.5^{\circ}$ to $29.6^{\circ}$ ) was attributed to highly disordered carbon and decrease in the sp2 $(\mathrm{C}-\mathrm{C})$ layers spacing in the carbonization process. The decrease in the intensity of the three peaks and the increase of the full width half maximum were due to the amorphous carbon structure of the prepared I-doped CDs. These results further indicated that these I-doped CDs with poor crystalline nature possessed heterogeneous multilayered structure, which was consistent with our previous reports about other CDs. ${ }^{28,29}$

\section{The X-ray attenuation and optical property of the I-doped CDs}

We then investigated the X-ray attenuation capacity of the I-doped CDs compared with the iodixanol (commercial contrast agents). As shown in Figure 4B, the CT image brightness increased with the I-doped CDs concentration, which was similar to the behavior of the iodixanol. At the iodine concentration of $0.1 \mathrm{mg} / \mathrm{mL}$ or above, the CT image was much brighter than those iodixanol under the same dosage of radiation. Further quantitative analysis data show that X-ray attenuation intensity of both I-doped CDs and the iodixanol increased with the mass concentration of radiodense element of the iodine. Nevertheless, the I-doped CDs exhibited much higher X-ray attenuation capacity than iodixanol under the same radiodense element concentrations $(0.1 \mathrm{mg} / \mathrm{mL}$ or above). The superior X-ray attenuation 

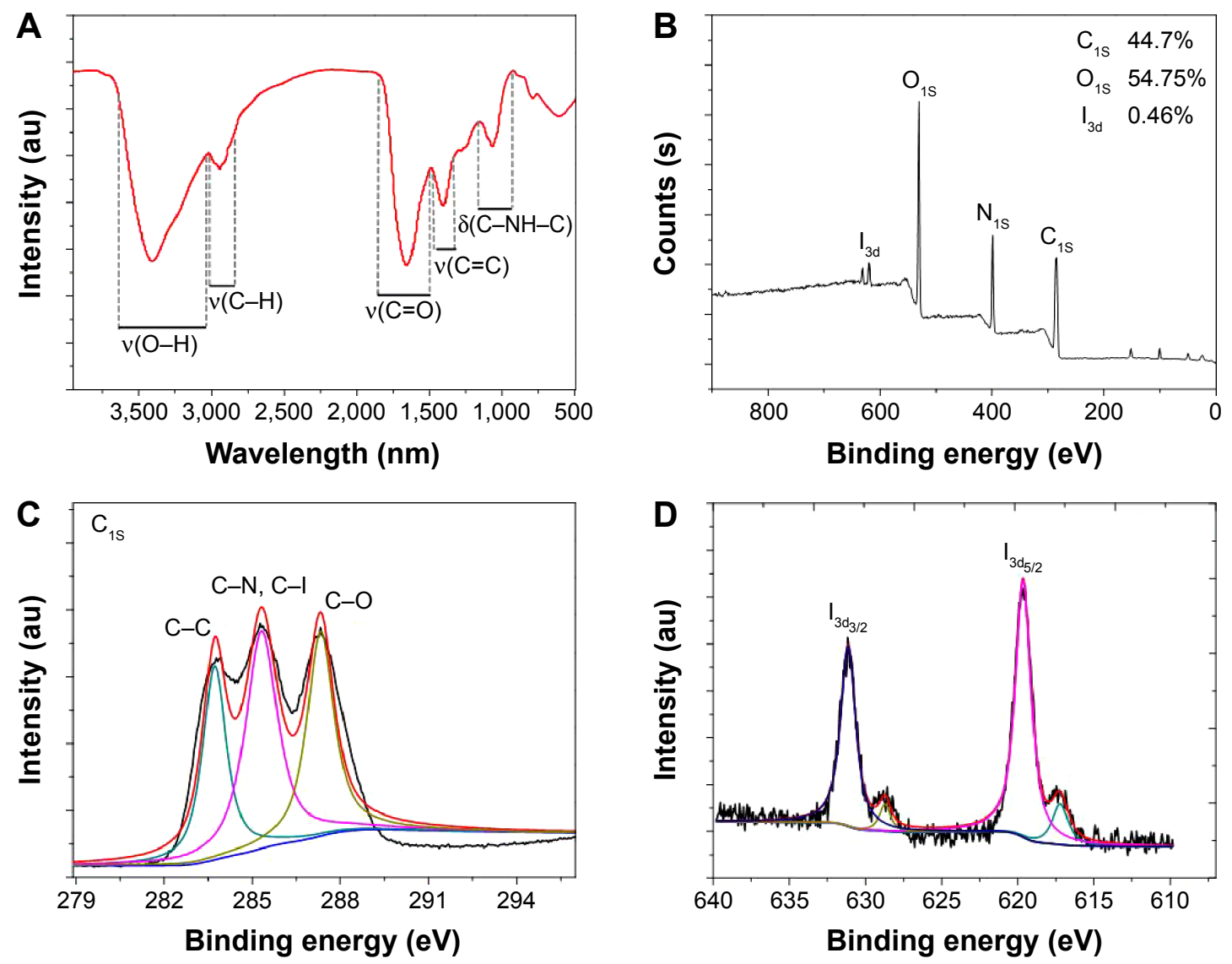

\begin{tabular}{|ll|}
\hline Raw intensity & Peak sum \\
Background & $-287.3 \mathrm{eV}$ \\
$-285.3 \mathrm{eV}$ & $-283.7 \mathrm{eV}$ \\
\hline
\end{tabular}

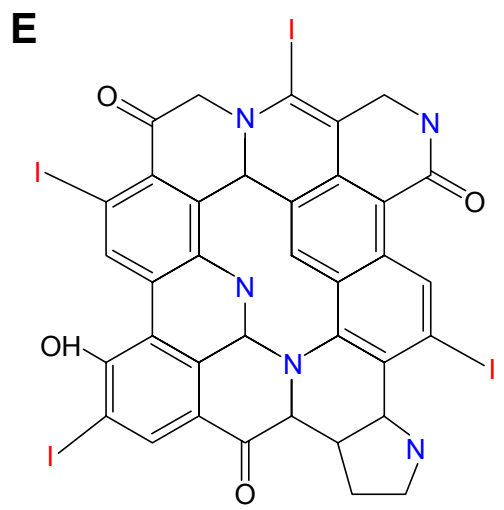
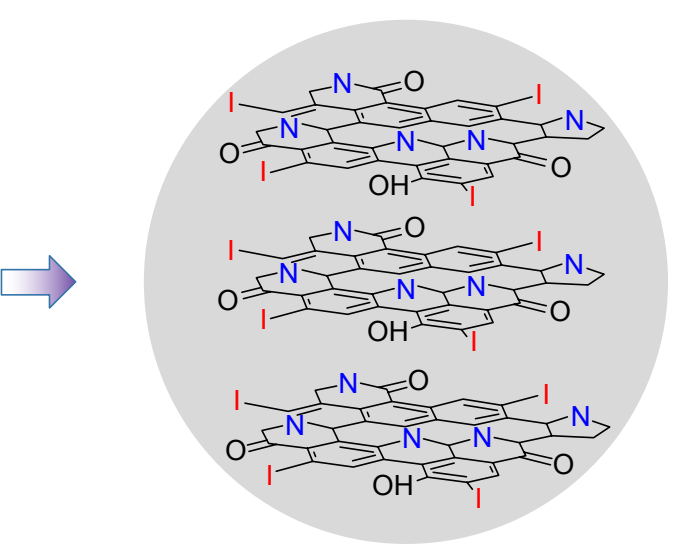

Figure 3 The chemical structure and composition of the I-doped CDs.

Notes: (A) The FTIR spectrum of I-doped CDs. (B) The full-scan XPS spectrum of I-doped CDs. (C) $C_{\text {IS }}$ spectrum. (D) I $_{3 \mathrm{~d}}$ spectrum. (E) A potential schematic illustration of doped iodine atom into the carbon particles.

Abbreviations: FTIR, Fourier transform infrared spectroscopy; I-doped CDs, iodine-doped carbon dots; XPS, X-ray photoelectron spectroscopy.

capacity endowed the I-doped CDs with more sensitive CT imaging applications.

The optical properties of the I-doped CDs were characterized using UV-Vis and PL spectrum (Figure 5). No obvious absorption peak could be observed in the UV-Vis spectrum (Figure 5A). The aqueous solution was pale yellow and transparent in daylight, but emitted bright blue fluorescence under UV excitation (inset, Figure 5A). The maximum emission at $475 \mathrm{~nm}$ was observed under $370 \mathrm{~nm}$ excitation. Additionally, the PL emission of the I-doped CDs, including 
A

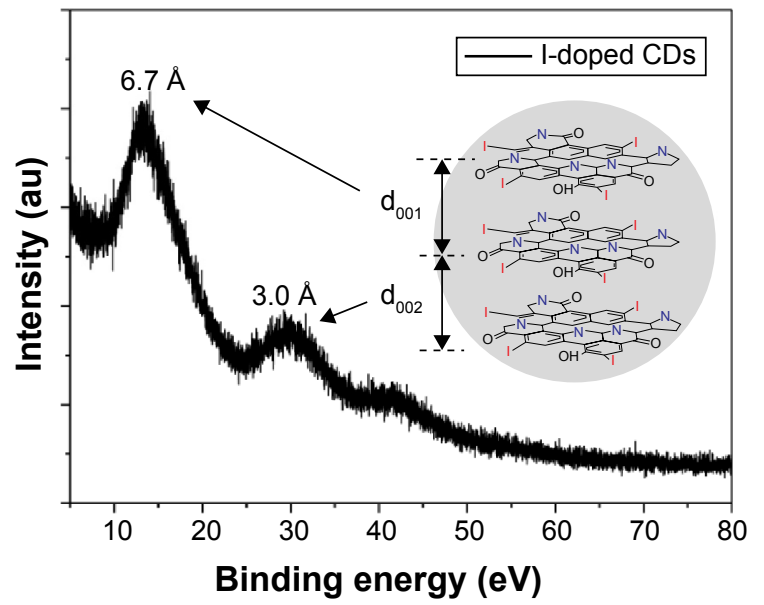

B

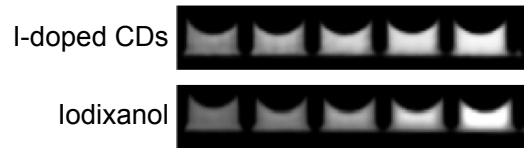

C

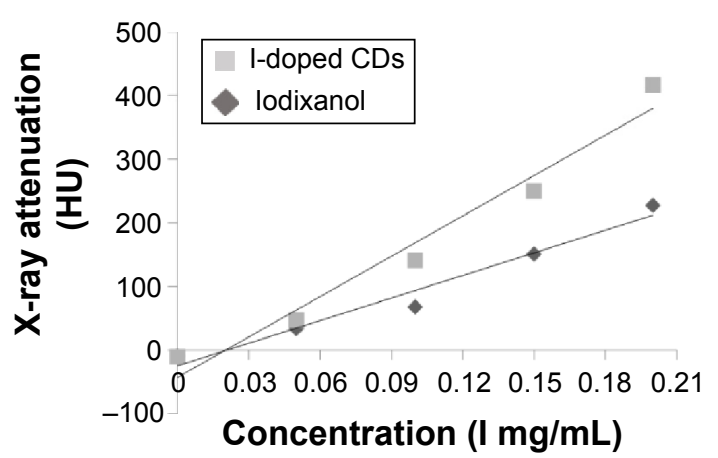

Figure $4 \times R D$ patterns and radio-opacity of the I-doped CDs.

Notes: (A) XRD patterns of the I-doped CDs. The inset represents the heterogeneous multilayer of the I-doped CDs, corresponding to characteristic diffraction peaks. (B) In vitro CT imaging of the l-doped CDs with different iodine concentrations from 0.05 to $0.2 \mathrm{mg} / \mathrm{mL}$. (C) The measured CT value of the five samples as a function of iodine concentration.

Abbreviations: XRD, X-ray diffraction; I-doped CDs, iodine-doped carbon dots; CT, computed tomography.
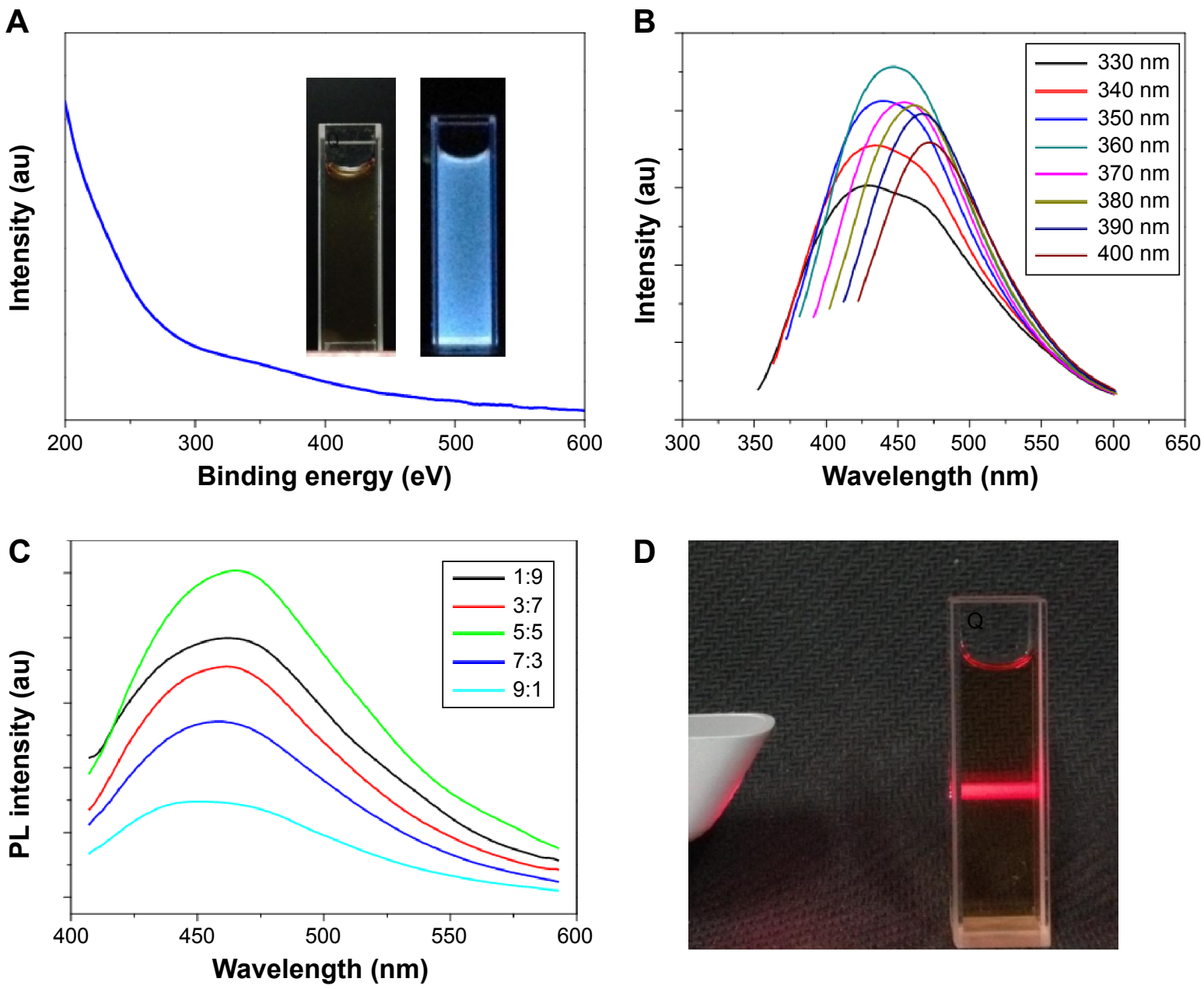

Figure 5 The optical properties of the I-doped CDs.

Notes: (A and B) UV-Vis absorbance and PL emission spectra of the I-doped CDs, respectively. The inset of (A) shows a diluted aqueous solution containing the as-prepared I-doped CDs. The PL emission spectra show the results of using progressively longer excitation wavelengths, from 330 to $400 \mathrm{~nm}$ in $10 \mathrm{~nm}$ increments. (C) The effect of molar ratio of glycine and iodixanol on the PL intensity. (D) The Tyndall effect exhibited by the I-doped CDs in aqueous solution.

Abbreviations: I-doped CDs, iodine-doped carbon dots; UV-Vis, ultraviolet-visible; PL, photoluminescence; Q, quartz. 
the wavelength and intensity in well-regulated mode within the excitation range of 330-510 nm, was wavelengthdependent excitation (Figure 5B). Although the emission peak gradually shifted from 450 to $550 \mathrm{~nm}$ with increasing excitation wavelength, the intensity of emission increased and reached a maximum at $390 \mathrm{~nm}$, beyond which the emission intensity gradually decreased. Figure 5C shows the effect of ratio of glycine and iodixanol on the PL of I-doped CDs. When the amount of iodine source equaled that of glycine (surface passivation), the PL intensity of I-doped CDs reached the maximum. The aqueous solution of I-doped CDs exhibited a long-term homogeneous phase without any noticeable precipitation at room temperature. To investigate the dispersibility in water, we used a beam of red light to illuminate the aqueous solution and observe the Tyndall effect clearly (as shown in Figure 5D). These results verified that the prepared I-doped CDs possessed superior fluorescent properties and dispersibility in aqueous solutions.

\section{The cytotoxicity and optical imaging of the I-doped CDs}

As potential biomedical probes, the good biocompatibility of the I-doped CDs is critical for their biomedical application. In order to validate the cytotoxicity in vitro, the effect of I-doped CDs on 4T1 cells viability was assessed by the MTS assay. As shown in Figure 6A, the I-doped CDs exhibited minimum cytotoxicity. Even at the concentration of $200 \mu \mathrm{g} / \mathrm{mL}$ and a 24-hours exposure time, the cell viability was approximately $90 \%$. It was worth noting that the I-doped CDs could be ready to enter into the cytoplasm and distribute around the nucleus after incubation for 4 hours. We found that the labeled cells emitted bright fluorescence due to the presence of I-doped CDs in cytosol (Figure 6B). Based on this capacity, we propose that the I-doped CDs can be used as potential fluorescence probes for real-time and long-term living cell imaging applications. Additionally, the live-cell fluorescent images showed that there were no morphological differences between the I-doped CD-treated
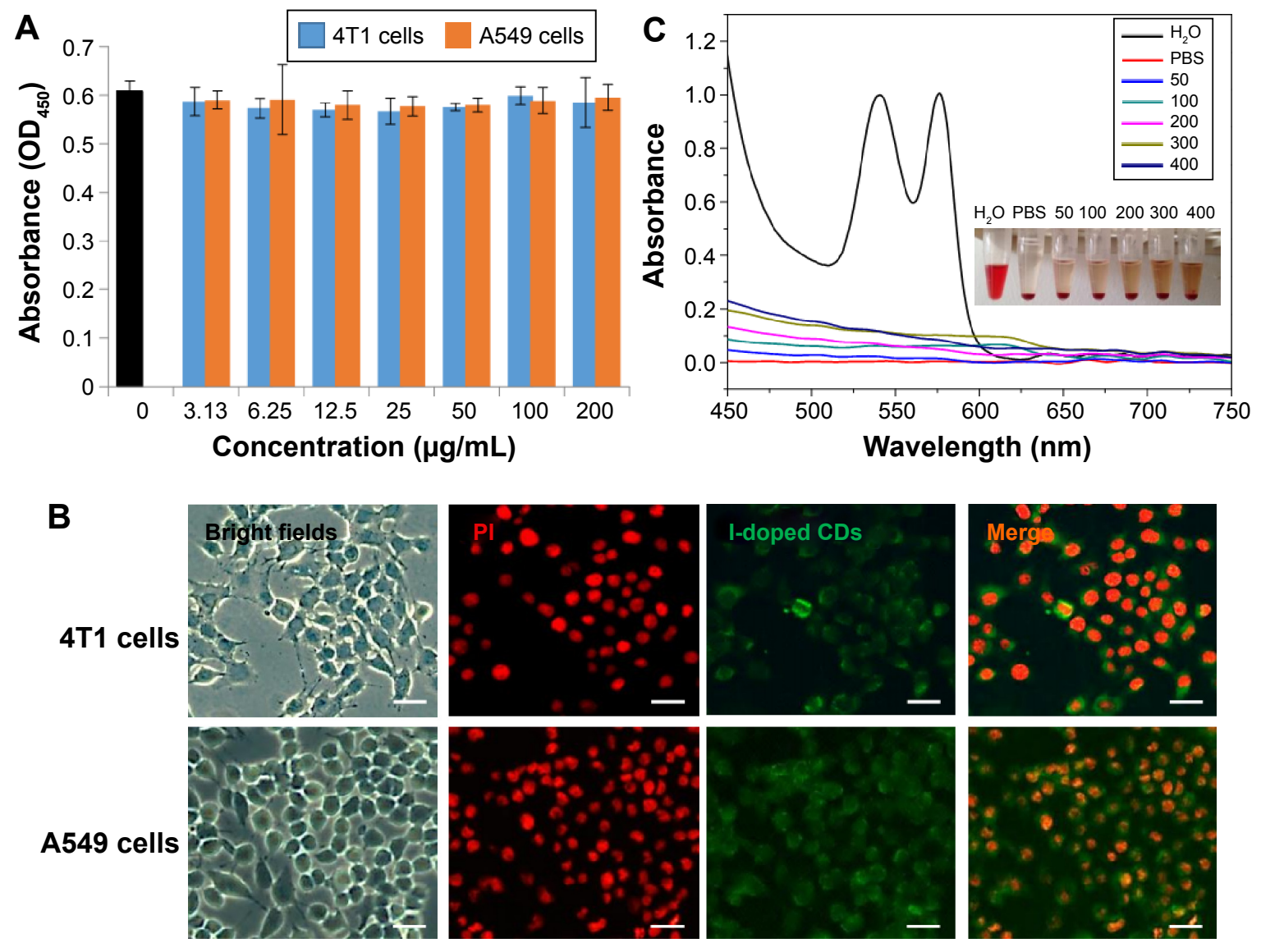

Figure 6 The charaterization of the I-doped CDs biocompatibility.

Notes: (A) Effect of I-doped CDs on the viability of 4TI and A549 cells using the MTS assay. (B) Laser scanning confocal microscopy images of 4TI and A549 cells stained with potassium iodide and I-doped CDs (scale bars $=50 \mu \mathrm{m})$. (C) Hemolytical activity of the I-doped CDs at different concentrations $(50,100,200,300$, and $400 \mu g / m L)$. PBS and water were used as negative and positive control, respectively.

Abbreviations: OD, optical density; I-doped CDs, iodine-doped carbon dots; PBS, phosphate buffer saline; MTS, 3-(4,5-dimethyl-thiazol-2-yl)-5-(3-carboxymethoxyphenyl)2-(4-sulfophenyl)-2H-tetrazolium. 
and the vehicle-treated cells. These results clearly suggested that the as-synthesized I-doped CDs had very low cytotoxicity and good biocompatibility.

The potential utility of the I-doped CDs as contrast agents for X-ray CT in vivo was evaluated using hemolysis assay. As shown in Figure 6C, we found hemolysis of RBC in the positive control. On the contrary, no obvious hemolysis phenomenon was observed after incubation with different concentrations from 50 to $400 \mu \mathrm{g} / \mathrm{mL}$ for 2 hours, which was similar to the negative normal saline. Compared to the negative control, the percentage of hemolysis at different concentrations of I-doped CDs was quantitatively evaluated based on the absorbance of the supernatant at $541 \mathrm{~nm}$. The results indicated that the hemolysis percentages of the I-doped CDs were all less than 3\% in the studied concentration range from 50 to $400 \mu \mathrm{g} / \mathrm{mL}$, which verified their favorable hemocompatibility.

\section{The biodistribution and the X-ray imaging of the I-doped CDs in vivo}

The possibility to use I-doped CDs as contrast agents for CT imaging in vivo was studied using Sprague Dawley rats as a model. After intravenous injection of I-doped CDs at a dose of $40 \mathrm{mg} / \mathrm{kg}$, the CT images were acquired through different scans. Compared with preinjection (Figure 7A), a great contrast enhancement in bladder was observed clearly using the three dimensional (3-D) CT imaging (as shown in Figure 7B). Figure 7C showed that the signal both in the kidney and bladder strongly increased at 10 minutes postinjection, which indicated I-doped CDs accumulation. These results were further confirmed through CT transverse scans at different levels (Figure 7D and E). To further investigate whether I-doped CDs can induce adverse effect (such as tissue damage, inflammation, or lesions) during the long-term postinjection, we conducted the histological assessment of the mouse susceptible organs (liver, heart, spleen, lung, and kidney). As shown in Figure 8, no obvious adverse effect associated with the administration of the I-doped CDs was observed compared with the control group. Based on the results described above, we proposed that the I-doped CDs were rapidly distributed throughout the body and excreted from the bladder.

Usually, large nanoparticles (more than $20 \mathrm{~nm}$ ) suffered from a high uptake by the reticuloendothelial system and macrophages and rapid accumulation in the mononuclear phagocyte system (eg, liver and spleen), resulting in a short circulation half-time and long-term toxicity issues. ${ }^{30,31} \mathrm{We}$ have demonstrated that the prepared I-doped CDs with ultrasmall size $(2.7 \mathrm{~nm})$ could be readily excreted by renal clearance, therefore avoiding nonspecific accumulation in the tissue of host. Importantly, ultrasmall size nanoparticles circulating in the blood might also increase the possibility of passive targeting in tumors by enhancing permeation and retention effect. ${ }^{32,33}$ Based on these results, we proposed that the I-doped CDs as ideal CT contrast agents had the potential to efficiently target the region of interest (eg, diseased area or tumor), after systemic administration, with unbound rapidly renal clearance from the host.

\section{Preinjection}

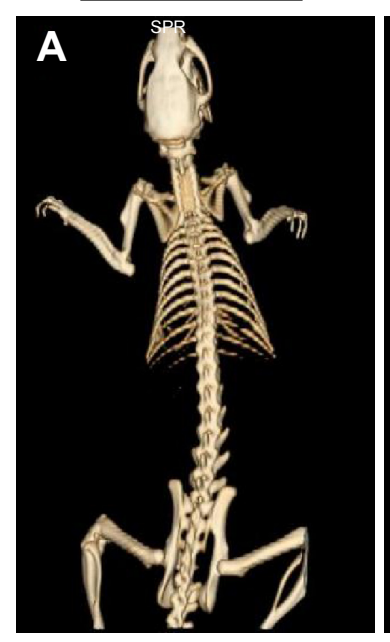

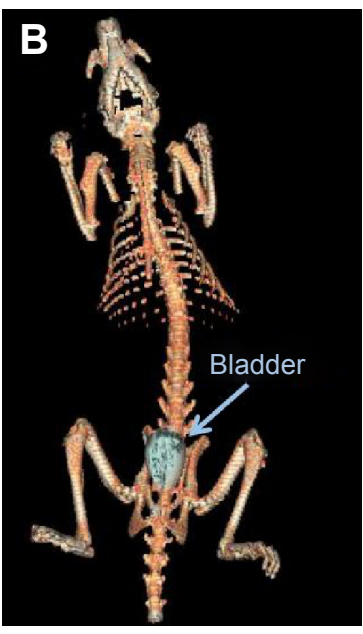

\section{Postinjection}

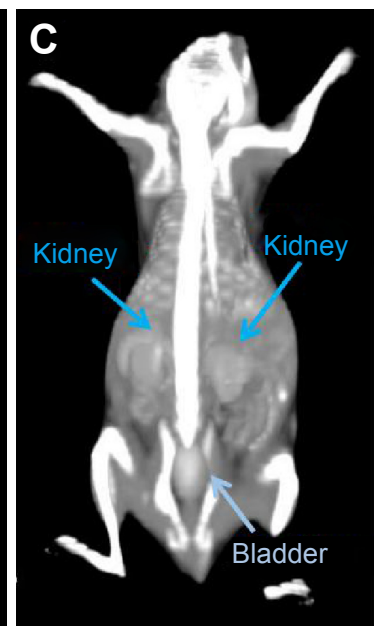

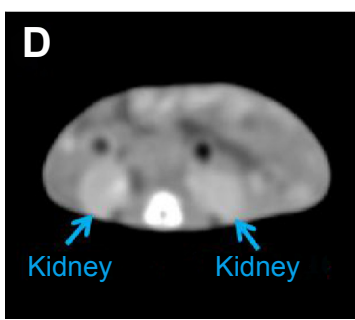

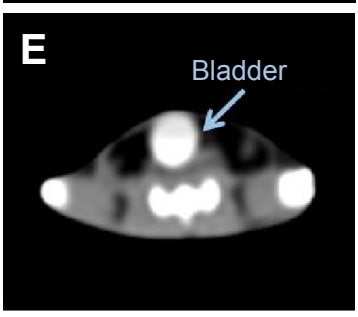

Figure 7 The X-ray CT imaging of I-doped CDs in vivo and in vitro.

Notes: 3-D X-ray CT imaging of rats before $(\mathbf{A})$ and after $(\mathbf{B})$ intravenous injection of I-doped CDs (40 mg/ $/ \mathrm{kg})$. In vivo CT imaging and analysis of rats after intravenous injection of I-doped CDs (C-E). Blue arrows represent kidney; gray arrows represent bladder.

Abbreviations: CT, computed tomography; I-doped CDs, iodine-doped carbon dots; 3-D, three dimensional. 

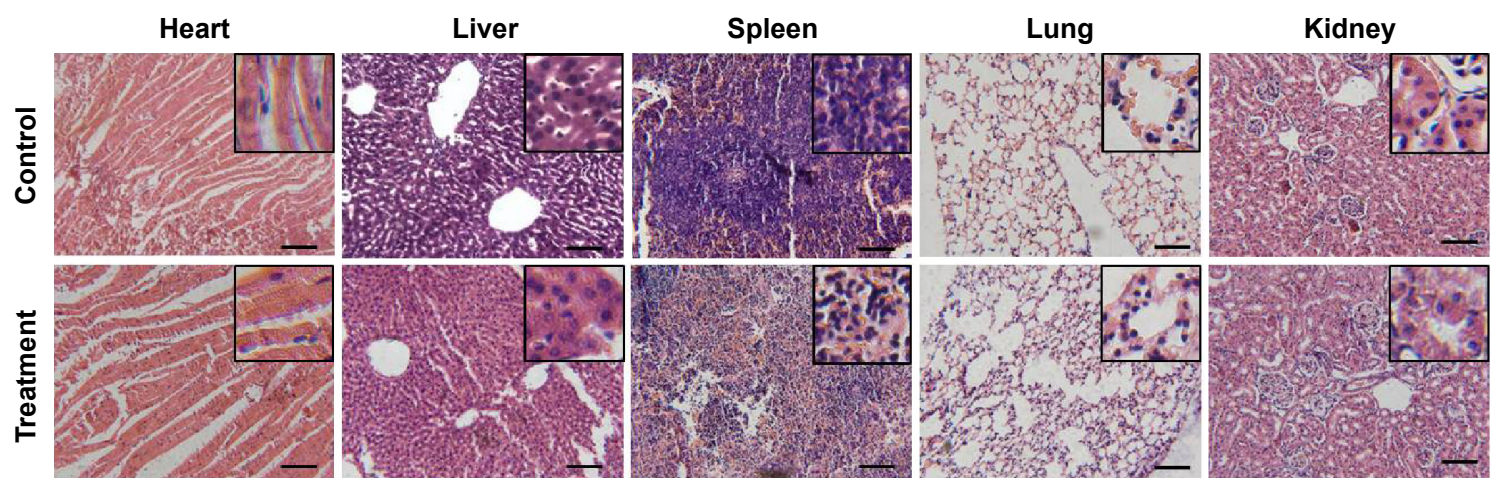

Figure 8 Histological changes in the organs of the rats 7 days after intravenous injection of I-doped CDs at the dose of $10 \mathrm{mg} / \mathrm{kg}$.

Notes: These organs were stained with H\&E and observed under a light microscope (scale bars $=50 \mu \mathrm{m}$ ). The inset figures represent the pictures were observed under high resolution.

Abbreviations: I-doped CDs, iodine-doped carbon dots; H\&E, hematoxylin and eosin.

\section{Conclusion}

We have developed dual-modal I-doped CDs for fluorescence and X-ray CT imaging by the HTC of glycine and iodixanol. Encapsulating iodine atoms into the carbon nanoparticles, the resultant I-doped CDs not only effectively overcame the side effect due to the intrinsic biological toxicity of iodine species, but also provided an attractive platform of introducing new physicochemical properties. The I-doped CDs exhibited favorable fluorescence properties and good biocompatibility, which were similar to previously reported carbon nanodots. Importantly, the high atomic number of iodine endowed these nanoparticles with strong X-ray attenuation capacity. The potential applications of I-doped CDs were strengthened by the fact that they could be efficiently excreted through renal clearance after systematic administration. In view of these merits, the novel I-doped CDs used as dual-modal probes have great potential applications in biomedical research and disease diagnosis.

\section{Acknowledgments}

The research was supported by the National Natural Science Foundation of China (Nos 81301316, 31200676), $\mathrm{PhD}$ Programs Foundation of Ministry of Education of China (No 20123227120008), China Postdoctoral Science Foundation (2013M540425, 2013M542520, 2014T70487, 2015M571705), Senior Talents Scientific Research Foundation of Jiangsu University (Nos 13JDG022, 11JDG113), and Zhenjiang City University Development Fund (No SH2013026).

\section{Disclosure}

The authors report no conflicts of interest in this work.

\section{References}

1. Kaittanis C, Shaffer TM, Ogirala A, et al. Environment-responsive nanophores for therapy and treatment monitoring via molecular MRI quenching. Nat Commun. 2014;5:3384.
2. Zhou Z, Huang D, Bao J, et al. A synergistically enhanced $\mathrm{T}_{1}-\mathrm{T}_{2}$ dualmodal contrast agent. Adv Mater. 2012;24(46):6223-6228.

3. Cho NH, Cheong TC, Min JH, et al. A multifunctional core-shell nanoparticle for dendritic cell-based cancer immunotherapy. Nat Nanotechnol. 2011;6(10):675-82.

4. Hong G, Lee JC, Robinson JT, et al. Multifunctional in vivo vascular imaging using near-infrared II fluorescence. Nat Med. 2012;18(12): 1841-1846.

5. Smet M, Langereis S, Bosch S, et al. SPECT/CT imaging of temperaturesensitive liposomes for MR-image guided drug delivery with high intensity focused ultrasound. J Control Release. 2013;169(1-2):82-90.

6. Li WP, Liao PY, Su CH, Yeh CS. Formation of oligonucleotide-gated silica shell-coated $\mathrm{Fe}_{3} \mathrm{O}_{4}$-Au core-shell nanotrisoctahedra for magnetically targeted and near-infrared light-responsive theranostic platform. J Am Chem Soc. 2014;136(28):10062-10075.

7. Stewart RC, Bansal PN, Entezari V, et al. Contrast-enhanced CT with a highaffinity cationic contrast agent for imaging ex vivo bovine, intact ex vivo rabbit, and in vivo rabbit cartilage. Radiology. 2013;266(1):141-150.

8. Gaedicke S, Braun F, Prasad S, et al. Noninvasive positron emission tomography and fluorescence imaging of CD133+ tumor stem cells. Proc Natl Acad Sci U S A. 2014;111(6):692-701.

9. Lee N, Choi SH, Hyeon T. Nano-sized CT contrast agents. Adv Mater. 2013;25(19):2641-2660.

10. Hainfeld JF, O'Connor MJ, Dilmanian FA, Slatkin DN, Adams DJ, Smilowitz HM. Micro-CT enables microlocalisation and quantification of Her2-targeted gold nanoparticles within tumour regions. Br J Radiol. 2011;84(1002):526-533.

11. Ashton JR, Clark DP, Moding EJ, et al. Dual-energy micro-ct functional imaging of primary lung cancer in mice using gold and iodine nanoparticle contrast agents: a validation study. PLoS One. 2014;9(2):88129.

12. Jakhmola A, Anton N, Anton H, et al. Poly-epsilon-caprolactone tungsten oxide nanoparticles as a contrast agent for X-ray computed tomography. Biomaterials. 2014;35(9):2981-2986.

13. Li X, Anton N, Zuber G, et al. Iodinated alpha-tocopherol nano-emulsions as non-toxic contrast agents for preclinical X-ray imaging. Biomaterials. 2013;34(2):481-491.

14. Myoung HO, Nohyun L, Hyoungsu K, et al. Large-scale synthesis of bioinert tantalum oxide nanoparticles for X-ray computed tomographyimaging and bimodal image-guided sentinel lymph node mapping. J Am Chem Soc. 2011;133:5508-5515.

15. Yu SB, Watson AD. Metal-based X-ray contrast media. Chem Rev. 1999;99(9):2353-2377.

16. Wang H, Zheng L, Peng C, Shen M, Shi X, Zhang G. Folic acid-modified dendrimer-entrapped gold nanoparticles as nanoprobes for targeted CT imaging of human lung adencarcinoma. Biomaterials. 2013;34(2): 470-480.

17. Cao L, Wang X, Meziani MJ, et al. Carbon dots for multiphoton bioimaging. J Am Chem Soc. 2007;129(37):11318-11319. 
18. Fan RJ, Sun Q, Zhang L, Zhang Y, Lu AH. Photoluminescent carbon dots directly derived from polyethylene glycol and their application for cellular imaging. Carbon. 2014;71:87-93.

19. Zhang R, Chen W. Nitrogen-doped carbon quantum dots: facile synthesis and application as a "turn-off" fluorescent probe for detection of $\mathrm{Hg}^{2+}$ ions. Biosens Bioelectron. 2014;55(0):83-90.

20. Dan L, Tang X, Pulli B, et al. Theranostic nanoparticles based on bioreducible polyethylenimine-coated iron oxide for reduction-responsive gene delivery and magnetic resonance imaging. Int J Nanomedicine. 2014; 9:3347-3361.

21. Du F, Zhang M, Li X, et al. Economical and green synthesis of bagasse-derived fluorescent carbon dots for biomedical applications. Nanotechnology. 2014;25(31):315702.

22. Du F, Li J, Hua Y, et al. Multicolor nitrogen-doped carbon dots for live cell imaging. J Biomed Nanotechnol. 2015;11(5):780-788.

23. Li Y, Zhao Y, Cheng H, et al. Nitrogen-doped graphene quantum dots with oxygen-rich functional groups. J Am Chem Soc. 2012;134(1):15-18.

24. Zhai X, Zhang P, Liu C, et al. Carbon nanoparticle-based fluorescent bioimaging probes. Chem Commun (Camb). 2013;48(64):7955-7957.

25. Li J, Zheng L, Cai H, et al. Polyethyleneimine-mediated synthesis of folic acid-targeted iron oxide nanoparticles for in vivo tumor MR imaging. Biomaterials. 2013;34(33):8382-8392.
26. Cai H, Li K, Shen M, et al. Facile assembly of Fe3O4@aunanocomposite particles for dual mode magnetic resonance and computedtomography imaging applications. J Mater Chem. 2012;22:15110-15120.

27. Wang Y, Duo F, Peng S, Jia F, Fan C. Potassium iodate assisted synthesis of titanium dioxide nanoparticles with superior water-dispersibility. J Colloid Interface Sci. 2014;430:31-39.

28. Zheng Z, Ai Z, Wang M, Zhang L. Doping iodine in CdS for pure hexagonal phase, narrower band gap, and enhanced photocatalytic activity. J Mater Res. 2011;26(5):710-719.

29. Du F, Yuan J, Zhang M, et al. Nitrogen-doped carbon dots with heterogeneous multi-layered structures. RSC Adv. 2014;4(71):37536-37541.

30. Du F, Jin X, Chen J, et al. Nitrogen-doped carbon dots as multifunctional fluorescent probes. J Nanopart Res. 2014;16:2720.

31. Gao J, Chen K, Xie R, et al. Ultrasmall near-infrared non-cadmium quantum dots for in vivo tumor imaging. Small. 2010;6(2):256-261.

32. Dong K, Ju E, Liu J, Han X, Ren J, Qu X. Ultrasmall biomoleculeanchored hybrid $\mathrm{GdVO}_{4}$ nanophosphors as a metabolizable multimodal bioimaging contrast agent. Nanoscale. 2014;6(20):12042-12049.

33. Zhou Z, Wang L, Chi X, et al. Engineered iron-oxide-based nanoparticles as enhanced T1 contrast agents for efficient tumor imaging. ACS Nano. 2013;7(4):3287-3296.
International Journal of Nanomedicine

\section{Publish your work in this journal}

The International Journal of Nanomedicine is an international, peerreviewed journal focusing on the application of nanotechnology in diagnostics, therapeutics, and drug delivery systems throughout the biomedical field. This journal is indexed on PubMed Central, MedLine, CAS, SciSearch $®$, Current Contents $\AA /$ Clinical Medicine,

\section{Dovepress}

Journal Citation Reports/Science Edition, EMBase, Scopus and the Elsevier Bibliographic databases. The manuscript management system is completely online and includes a very quick and fair peer-review system, which is all easy to use. Visit http://www.dovepress.com/ testimonials.php to read real quotes from published authors. 